\title{
Mulibrey nanism
}

INSERM

\section{Source}

INSERM. (1999). Orphanet: an online rare disease and orphan drug data base. Mulibrey nanism. ORPHA:2576

A rare developmental defect during embryogenesis characterized by growth delay and multiorgan manifestations. 